\title{
Math Suggestion: Uma Ferramenta de Recomendação de Objetos de Aprendizagem Fundamentada nos Princípios das Avaliações de Autoeficácia e Análise de Desempenho
}

\author{
Vinícius A. Silva ${ }^{1}$, Hiran N. M. Ferreira ${ }^{1}$, Agmar de A. Torres ${ }^{1,2}$, Fredy C. Rodrigues ${ }^{1}$ \\ ${ }^{1}$ Instituto Federal de Educação, Ciência e Tecnologia do Sul de Minas Gerais \\ (IFSULDEMINAS) - Passos/MG, Brasil \\ ${ }^{2}$ Faculdade de Computação - Universidade Federal de Uberlândia (UFU) \\ Uberlândia/MG, Brasil
}

\begin{abstract}
This paper presents Math Suggestion, a tool that aims to enable the elaboration and application of a diagnostic evaluation using self-efficacy and performance to recommends Learning Objects (LOs) based on the abilities and limitations of the students. For the recommendation, the tool uses the concepts of pedagogical approaches based on the theory of self-efficacy and performance. In addition to the description of the tool, this paper also presents experiments, applied to incoming students from three higher courses, with the purpose of evaluating the utility perception of the tool. Results showed that this proposal can positively impact students' learning and also influence their behavior.
\end{abstract}

Resumo. Este trabalho apresenta a Math Suggestion, uma ferramenta que tem como objetivo possibilitar a elaboração e aplicação de uma avaliação diagnóstica utilizando instrumentos de autoeficácia e de desempenho e em seguida recomendar objetos de aprendizagem (OAs) com base nas capacidades e limitações dos estudantes. Para a recomendação, a ferramenta utiliza os conceitos de abordagens pedagógicas fundamentados na teoria de autoeficácia e desempenho. Além da descrição da ferramenta, este artigo também apresenta experimentos, aplicados a estudantes ingressantes de três cursos superiores, com o objetivo de avaliar a percepção de utilidade da ferramenta. Resultados mostraram que esta proposta pode impactar positivamente na aprendizagem dos estudantes e também influenciar seus comportamentos.

\section{Introdução}

A disciplina de matemática tanto no Ensino Fundamental quanto no Médio possui cargas horárias maiores em relação a outras disciplinas, e ainda assim há, de um modo geral, pouca interação entre estudante e a disciplina [Rodrigues 2017]. Vale destacar ainda que a matemática ensinada no Ensino Fundamental e Médio é utilizada como requisito básico no Ensino Superior em cursos de ciências exatas, influenciando todo o processo educacional e acadêmico do estudante. Conforme [Tomás da Silva et al. 2017], a matemática tem sido categorizada como um filtro para o acesso a carreiras científicas e tecnológicas no Brasil, resultando em reprovações e desistências nos períodos iniciais dos cursos.

Muitos fatores têm sido estudados nos processos de aprendizagem matemática, como aspectos socioeconômicos, cognitivos e afetivos dos estudantes [Tomás da Silva et al. 2017]. Dentre esses fatores, as crenças de autoeficácia ma- 
temática, que se referem às crenças pessoais em resolver problemas que envolvem matemática, têm tido um papel de destaque. Pesquisas também indicam que a crença de autoeficácia é um dos motivos pelos quais indivíduos com as mesmas habilidades e conhecimentos apresentam desempenhos distintos, de forma que quanto maior as crenças de autoeficácia em relação a um determinado conteúdo, maior a probabilidade de um bom desempenho escolar [Brito and Souza 2015].

Nesse contexto, alternativas para suprir a necessidade de diminuir o déficit de conteúdo dos estudantes têm sido alvo de pesquisas. Dentre as ações, houve um aumento na emergência de tecnologias digitais da informação e comunicação direcionadas a auxiliar nos processos educacionais. Pesquisas recentes revelam que a utilização destas proporcionou o surgimento de novas técnicas de ensino e um aumento considerável na performance dos alunos [Bray and Tangney 2017].

No que se refere a tecnologias digitais, muitos trabalhos demonstram que sistemas de recomendação oferecem vantagens no contexto educacional [Aguiar et al. 2015]. Os sistemas de recomendação atuam como filtros de informações para apresentar recursos educacionais necessários e interessantes aos alunos, articulando os resultados da aplicação a metadados e informações fornecidas pelos usuários.

Considerando o que foi exposto, este trabalho apresenta a ferramenta Math Suggestion, um ambiente computacional fundamentado nas teorias das crenças de autoeficácia matemática [Bandura 1986, Brito and Souza 2015], que tem como principal funcionalidade a análise diagnóstica dos níveis de conhecimento dos estudantes para, a partir disso, recomendar conteúdo pedagógico adequado às suas capacidades e dificuldades. A ferramenta identifica o perfil de cada estudante e, com base no seu nível de conhecimento, recomenda diferentes Objetos de Aprendizagem (OAs) relacionados aos conteúdos em que os estudantes possuem mais déficits. Além da descrição da ferramenta, este trabalho realizou experimentos com turmas ingressantes de cursos superiores presenciais com o intuito de avaliar o impacto da utilização da ferramenta no comportamento dos estudantes. Um total de 84 estudantes de três diferentes cursos participaram dos experimentos.

\section{Fundamentação Teórica}

Esta seção apresenta brevemente os conceitos da crença de autoeficácia e desempenho e de sistemas de recomendação de objetos de aprendizagem.

\subsection{Crença de autoeficácia e desempenho}

De acordo com os pressupostos da Teoria Social Cognitiva de Albert Bandura [Bandura 1986] a maneira pela qual os indivíduos resolvem problemas é fortemente influenciado por suas crenças pessoais em relação a sua capacidade de resolver determinadas ações de curso específicas [Dobarro and Brito 2010]. Ao avaliar o desempenho dos estudantes na disciplina de Matemática, [Pajares and Miller 1994] revelaram que as crenças de autoeficácia constituem um importante elemento que determina a quantidade de tempo e esforço despendido na realização de um problema.

O conceito de autoeficácia foi definido por Bandura [Bandura 1997] como a crença na própria capacidade de organizar e executar cursos de ações requeridas para produzir determinadas realizações. Trata-se, portanto, de uma autoavaliação de competência 
realizada pelo estudante no que diz respeito a sua capacidade de realizar uma tarefa ou problema em específico.

De acordo com [Dobarro and Brito 2010], as crenças pessoais de eficácia constituem o principal fator do fazer humano, pois por meio de crenças é que as pessoas avaliam se elas são ou não capazes de fazer e produzir resultados. Pesquisas também indicam que a crença de autoeficácia é um dos motivos pelas quais indivíduos com as mesmas habilidades e conhecimentos apresentam desempenhos distintos em uma determinada tarefa [Dobarro and Brito 2010].

Pesquisas sobre crenças de autoeficácia realizadas por [Lent et al. 1984, Pajares 1996, Pietsch et al. 2003] apontam para uma estreita relação entre este constructo mental e o desempenho acadêmico em matemática. Nesse sentido, quanto maior as crenças de autoeficácia em relação a uma determinada tarefa, maior a probabilidade de um bom desempenho escolar [Brito and Souza 2015].

Nesse contexto, [Barros 1996] revela que a autoeficácia está relacionada a atribuições de causalidade para o sucesso ou fracasso na disciplina de matemática, podendo assim ser tomada como um forte elemento preditivo do desempenho escolar em determinada tarefa.

\subsection{Sistemas para Recomendação de Objetos de Aprendizagem}

Na literatura é possível encontrar diversas abordagens para recomendação de OAs. Tais abordagens se diferem, principalmente, pela arquitetura do modelo, pela abordagem pedagógica empregada ou, até mesmo, pelo contexto de recomendação.

Alguns trabalhos buscaram implementar abordagens com base em teorias pedagógicas aplicadas a diferentes grupos de estudantes [Dorça et al. 2016, Syed et al. 2017, Zia and Usman 2018]. No trabalho de [Syed et al. 2017] foi proposto um ambiente para recomendação que baseia-se no comportamento de grupos de estudantes para realização das recomendações. Já no trabalho de [Zia and Usman 2018] foi proposto um ambiente para recomendação que baseia-se na teoria dos jogos para propor uma recomendação específica com base no comportamento do estudante. Por outro lado, o trabalho de [Dorça et al. 2016], baseou-se na teoria de Estilos de Aprendizagem para recomendar conteúdo adaptado às características de aprendizagem de cada estudante. Todos esses trabalhos diferem da abordagem aqui proposta por não utilizarem o comportamento cognitivo e abordagens de avaliação do estudante como métricas para recomendação.

Alguns trabalhos empregam abordagens computacionais com diferentes objetivos. No trabalho de [Rezende et al. 2015] foi descrito um ambiente para recomendação de OAs que utiliza uma ontologia para a modelagem do perfil e do contexto do estudante em um ambiente e-learning. Em [Pukkhem 2013] foi proposto um framework semântico para personalização em e-learning baseado no estilo de aprendizagem do estudante. Esse framework emprega ontologias para que computadores sejam capazes de interpretar e processar objetos de aprendizagem em um sistema de recomendação. Em [Imran et al. 2016] foi proposto um ambiente que considera a distância entre o nível de aprendizagem dos diferentes estudantes afim de propor uma recomendação mais efetiva. Diferente da abordagem aqui proposta, tais ambientes não utilizam instrumentos de avaliação e feedbacks para compor o ambiente de recomendação. 


\section{Arquitetura da Math Suggestion}

De acordo com [Brito and Souza 2015] o processo de ensino-aprendizagem de matemática deve levar em consideração, além do desenvolvimento de habilidades, a construção de autopercepções favoráveis do estudante em relação aos conteúdos.

Em vista disto, a ferramenta Math Suggestion foi elaborada com base no princípio das crenças de autoeficácia e desempenho, fazendo uso desses instrumentos para recomendar OAs mais voltados para as necessidades dos estudantes. A ferramenta possui dois perfis de usuário, que são professor e estudante, possibilitando:

- Ao estudante:

- Responder os instrumentos de avaliação (autoeficácia e/ou desempenho) em uma plataforma digital;

- Conhecer o nível de conhecimento e suas autopercepções sobre os conteúdos (conforme instrumentos elaborados pelo professor);

- Receber os OAs mais indicados de acordo com a sua dificuldade.

- Ao professor:

- Criar instrumentos para realizar uma análise diagnóstica levando-se em consideração a autopercepção e desempenho dos estudantes em relação a um determinado conteúdo;

- Visualizar graficamente o nível de conhecimento dos estudantes, de forma individualizada ou por turma;

- Disponibilizar OAs para os estudantes, de forma que a ferramenta irá avaliar e recomendar os OAs mais indicados individualmente;

- Acompanhar as interações dos estudantes com os OAs indicados.

A arquitetura da Math Suggestion (Figura 1) é composta, fundamentalmente, por cinco módulos: Módulo de Administração (perfil administrador), Módulo de Criação dos Instrumentos Avaliativos, Módulo de Análise Diagnóstica (perfil professor), Módulo de Objetos de Aprendizagem e Módulo de Recomendação. Para a implementação do sistema foi utilizado o framework MVC PHP CodeIgniter, HTML5, Bootstrap, javascript e Jquery.

A Figura 1 apresenta em detalhes a arquitetura do Math Suggestion.

Em relação ao funcionamento da ferramenta, o administrador cria (Figura 1-A) usuários para os professores mediante solicitação por e-mail e comprovação de docência. $\mathrm{O}$ administrador também mantém as categorias referentes aos conteúdos de matemática abordados pela ferramenta. Após o cadastro, o professor consegue elaborar (Figura 1-B) os instrumentos de avaliação com questões objetivas com base nas crenças de autoeficácia e desempenho. Cada questão do instrumento está relacionada a uma categoria (Figura 1C). As questões do instrumento de autoeficácia, proposto por [Bandura 1986], questionam o estudante sobre a sua autopercepção sobre uma determinada categoria/conteúdo de matemática (por exemplo: estou seguro em resolver problemas que envolvem relações trigonométricas? As respostas possíveis são: Discordo totalmente, Discordo, Concordo, Concordo Totalmente). O professor também deve inserir os objetos de aprendizagem (Figura 1-D) informando qual categoria e o nível do objeto inserido, como mostra a Figura 2.

O método de recomendação do sistema utiliza essas informações para disponibilizar os OAs de acordo com as respostas dos usuários nos instrumentos. Se o estudante informar que não possui segurança em relação a um determinado conteúdo (autoeficácia) 


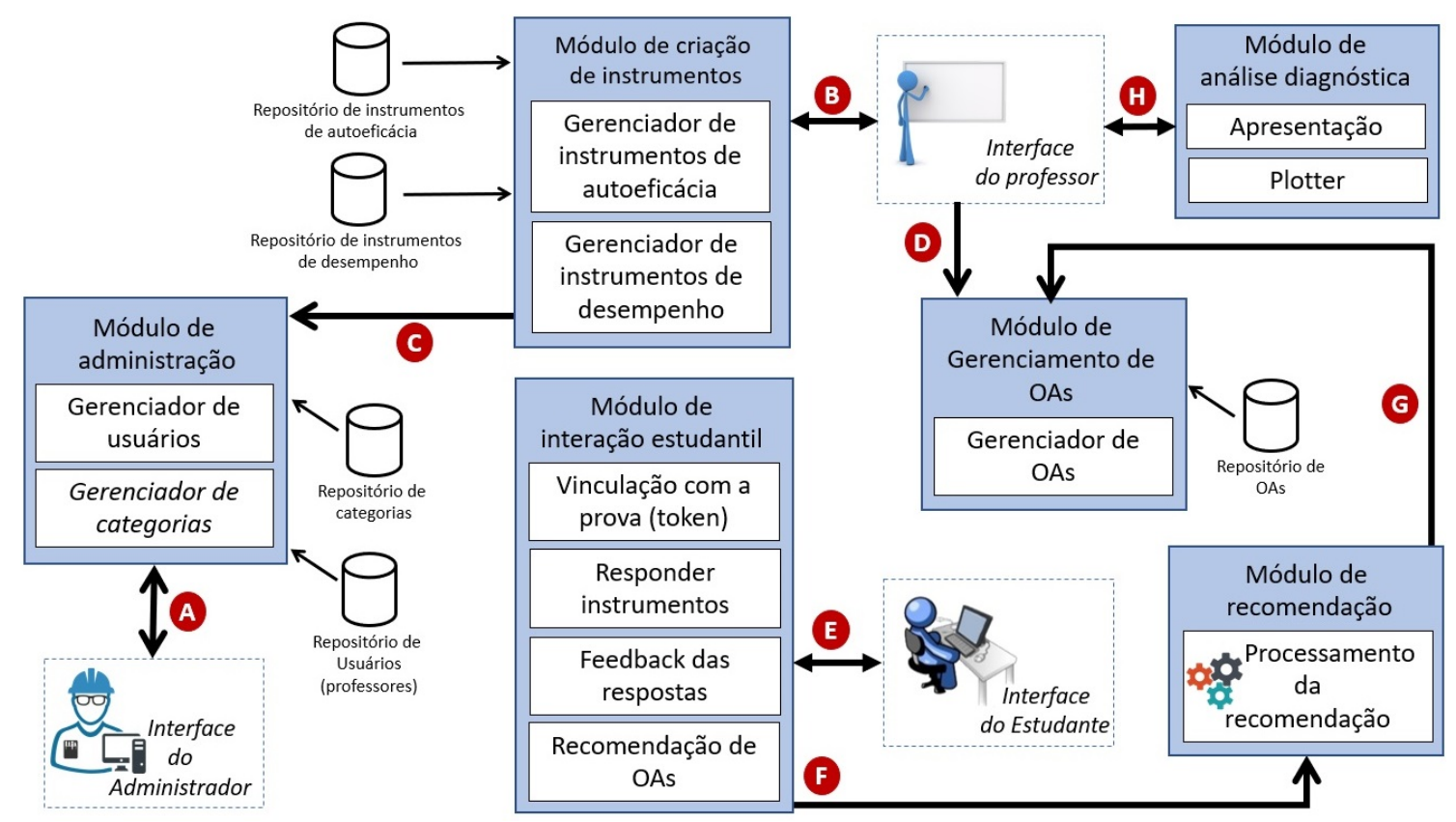

Figura 1. Arquitetura do Math Suggestion

ou errar a questão sobre a categoria (desempenho), o sistema recomenda OAs sobre o conteúdo. É importante destacar que neste momento é criado um perfil para cada estudante, permitindo assim, registrar suas capacidades individualizadas.

O estudante inicialmente deve realizar um cadastro na ferramenta Math Suggestion. Feito isso, o usuário será redirecionado para a página de vínculo de avaliação na qual deverá digitar o token da avaliação criada pelo professor. Após essa etapa de vínculo da avaliação o estudante é redirecionado para responder inicialmente ao instrumento com as questões de autoeficácia (Figura 1-E). Em um segundo momento, os estudantes resolvem também o instrumento de desempenho. Após fazer as questões dos instrumentos, o estudante recebe um feedback em relação aos erros e acertos. É importante destacar que o professor tem acesso (Figura 1-H) a gráficos e relatórios com informações referentes

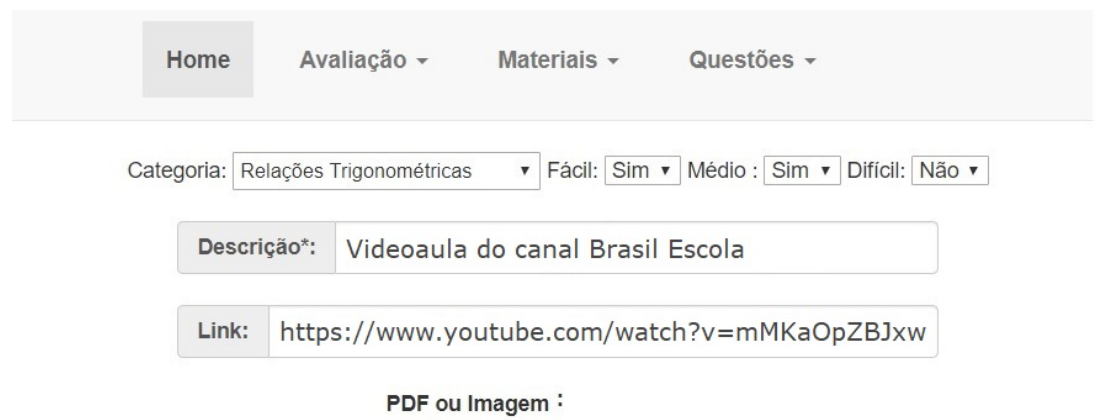

Escolher arquivo Nenhum arquivo selecionado 
às percepções (autoeficácia) e desempenho dos estudantes. As informações podem ser acessadas para uma turma (Figura 3) ou para um estudante específico (Figura 4).

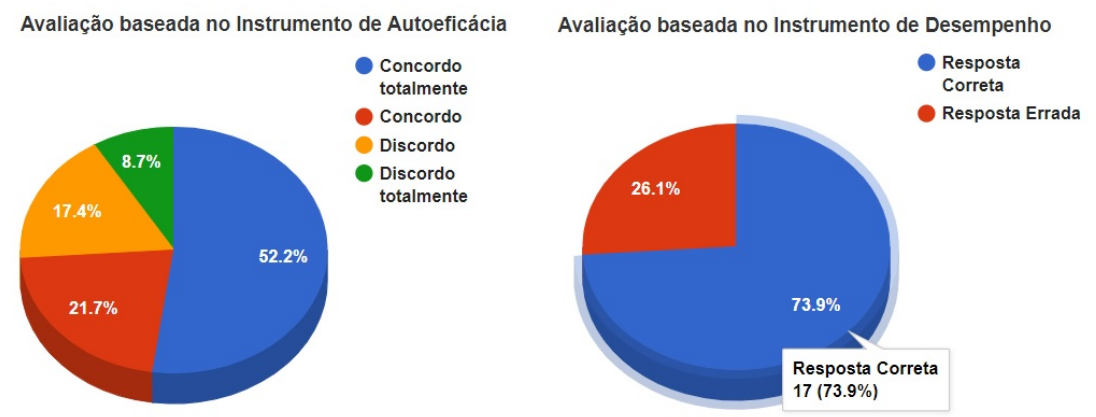

Figura 3. Gráfico geral de respostas de um estudante específico para os instrumentos de autoeficácia e desempenho
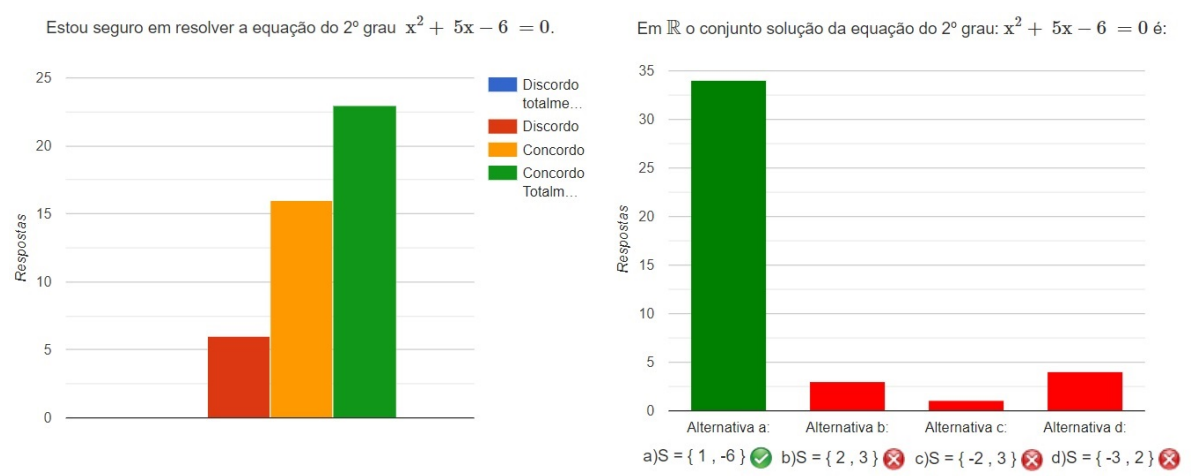

Figura 4. Gráfico das respostas de uma turma para uma questão específica

Em seguida, o sistema recomenda OAs (Figura 1-F) automaticamente para o estudante conforme o resultado dos instrumentos preenchidos. Vale destacar que essa recomendação é baseada no perfil criado anteriormente, ou seja, cada estudante recebe recomendações individualizadas de acordo com suas capacidades e limitações. A Figura (Figura 5) mostra a tela de recomendação de OAs.

Os critérios para a recomendação foram definidos em reuniões com professores da área de matemática. A ferramenta utiliza uma filtragem baseada em categoria e nível de dificuldade das questões e materiais de apoio. Esse método de filtragem pode ser considerado como uma personalização do método baseado em conteúdo uma vez que, o método baseado em conteúdo utiliza dados do usuário para fazer as recomendações necessárias. $\mathrm{O}$ algoritmo de recomendação propicia materiais de apoio baseado no nível de dificuldade das questões, evitando assim que discentes tenham acesso a materiais mais complexos que o seu discernimento, ou materiais sobre os quais ele já possua conhecimentos.

Os OAs recomendados para os discentes são utilizados como proposta de intervenção, estimulando o estudante a estudar os conteúdos que tem dificuldade. Os relatórios diagnósticos disponibilizados para o professor auxiliam no processo de tomada de decisão para o desenvolvimento das aulas por meio de revisões de acordo com informações colhidas com os instrumentos de avaliação. 

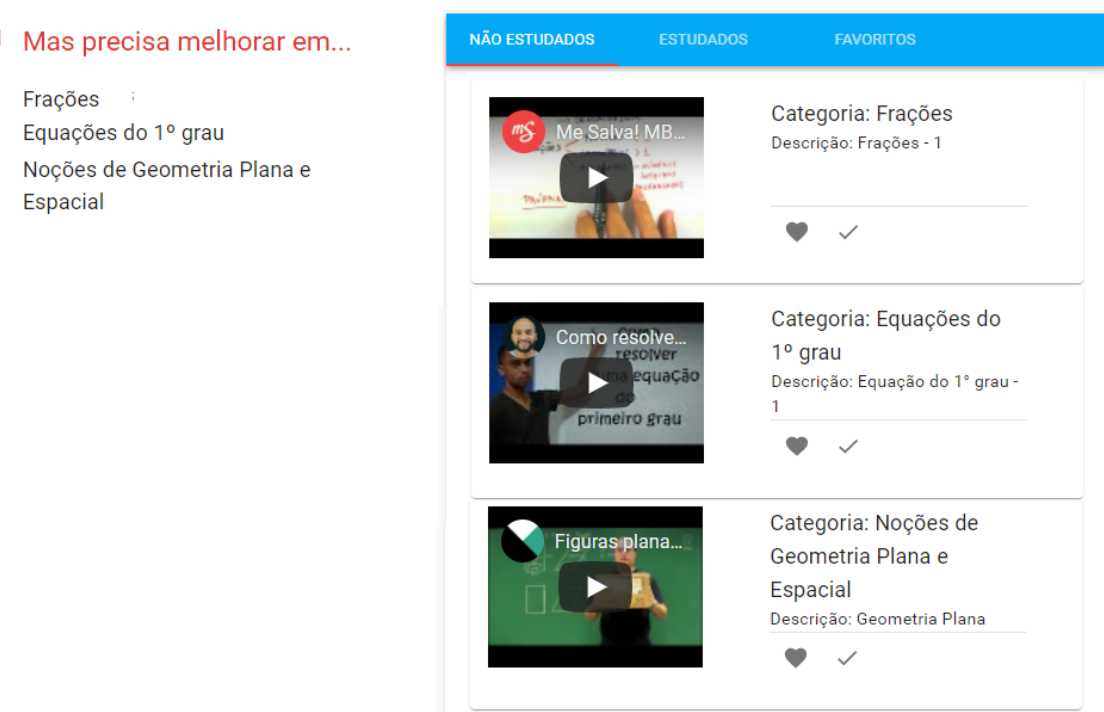

Figura 5. Tela de Recomendação de OAs

\section{Experimento e Resultados}

Buscando analisar o impacto causado pela ferramenta Math Suggestion na satisfação e no comportamento dos estudantes, foi conduzido um experimento qualitativo com o intuito de verificar se o fato dos estudantes conseguirem saber o seu nível de conhecimento prévio em determinada disciplina e ter conteúdo educacional adequado às suas dificuldades influenciou positiva ou negativamente no seu comportamento (motivação, competitividade, interesse pelos estudos, entre outros fatores).

\subsection{Método Aplicado}

O experimento foi aplicado a três turmas ingressantes na disciplina de Fundamentos de Matemática em uma instituição pública de ensino situada no sul do Estado de Minas Gerais. No total, foram respondidos 41 questionários na turma do curso de Bacharelado em Ciência da Computação, 25 questionários na turma do curso de Licenciatura em Matemática e 18 questionários na turma do curso de Bacharelado em Administração, totalizando 84 questionários.

No início da disciplina, o professor apresentou a ferramenta Math Suggestion, demonstrando suas funcionalidades e explicando como seria sua utilização. O objetivo foi permitir que os estudantes utilizassem a ferramenta inicialmente para que ela obtivesse os níveis de conhecimento de cada um, e a partir disso, fosse criado o perfil individual de cada estudante. Após isso, a ferramenta já estava preparada para fornecer recursos adaptados às habilidades e dificuldades de cada estudantes individualmente.

Depois da utilização da ferramenta os estudantes responderam ao questionário proposto. Este apresentava, em sua maioria, perguntas classificadas em uma escala do tipo Likert de sete pontos (discordo totalmente a concordo totalmente). As perguntas foram classificadas em dois grupos: o primeiro relativo à percepção de utilidade das funcionalidades disponibilizadas pela plataforma e o segundo relativo à percepção de alteração no comportamento do estudante durantes seus estudos.

Além dos dois grupos apresentados anteriormente também foi disponibilizada 
uma questão discursiva com o intuito de oferecer uma avaliação mais subjetiva, na qual foi possível observar não somente a percepção sobre utilidade e satisfação na utilização da ferramenta, como também foi possível identificar pontos fracos e fortes das funcionalidades ${ }^{1}$.

\subsection{Resultados e Discussão}

O foco principal deste experimento foi verificar critérios relativos à satisfação, perspectiva de utilidade e o quanto os estudantes podem alterar o seu comportamento quando utilizam uma ferramenta de recomendação de conteúdo educacional. Neste sentido, como o experimento realizado incluiu questões descritivas, foi necessária uma avaliação qualitativa para uma melhor interpretação dos resultados. Assim, foi possível identificar que, em todas as questões analisadas relativas à percepção de utilidade, houve maiores concentrações de respostas entre os valores de concordância (Figura 6-A).

(A)

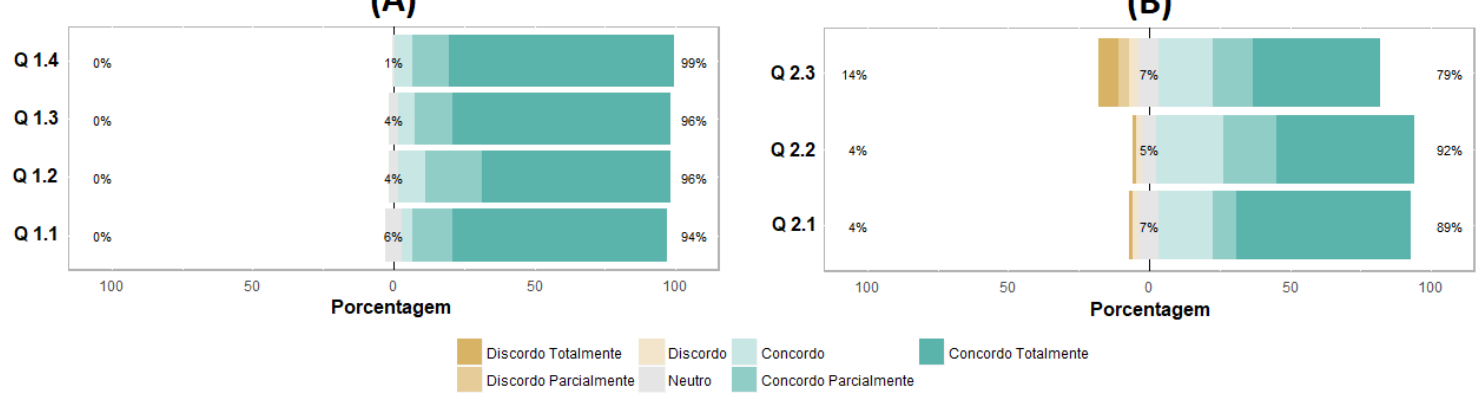

Figura 6. Grau de concordância sobre a percepção de utilidade.

Para a Questão 1.1 foi verificado se os estudantes acreditam que o fato de poder identificar seus conhecimentos prévio pode influenciar positivamente a sua aprendizagem. Neste caso, 94\% dos estudantes responderam que acredita que a ferramenta pode influenciar de forma significativa no aprendizado. Na Questão 1.2, 96\% dos estudantes responderam que acreditam que a ferramenta Math Suggestion é um importante mecanismo para auxiliar no desenvolvimento da disciplina. Na Questão $1.396 \%$ dos estudantes responderam que gostaram de utilizar a ferramenta e por fim, $99 \%$ dos estudantes disseram acreditar que a recomendação de conteúdos educacionais baseados em ferramenta de auto eficácia pode influenciar positivamente na aprendizagem em uma disciplina (Questão 1.4).

Além da perspectiva de utilidade o experimento também buscou avaliar o quanto os estudantes acreditam que a Math Suggestion pode alterar o comportamento daqueles que utilizam a ferramenta. Neste caso, foi possível observar, claramente, maiores concentrações de respostas entre os valores positivos (Figura 6-B).

Foi possível constatar por meio das respostas dos questionários que os estudantes acreditam que o fato de conhecerem previamente suas limitações e capacidades pode sim, de certa forma, alterar o seu comportamento da disciplina (Questão 2.1). Também foi possível constatar por meio da questão 2.2 que tal ferramenta possui a capacidade de deixar os estudantes mais motivados e consequentemente, mais interessados com os estudos. Quando considerada a capacidade do estudante se sentir mais competitivo, 79\%

\footnotetext{
${ }^{1}$ O questionário completo está disponível em: https://forms.gle/8SvzeRnR8dri7isB6
} 
dos estudantes disseram acreditar que a ferramenta pode prover tal característica. Em todo o questionário, essa foi a pergunta que foi obtida uma maior discordância por parte dos estudantes. Acredita-se que isso se dá pois a ferramenta não incentiva a interação entre estudantes e somente entre estudante e material didático.

A Figura 4.2 exibe uma síntese dos resultados, mostrando o percentual de resposta para cada uma das escalas likert além da mediana e do desvio padrão de cada uma das perguntas. É importante destacar que para todas as questões houve maior concentração de valores considerados positivos tanto em questões relacionadas com a perspectiva de utilidade quanto a perspectiva de alteração de comportamento. Esses resultados mostram que os estudantes acreditam que a ferramenta tem um potencial importante para a melhoria no aprendizado.

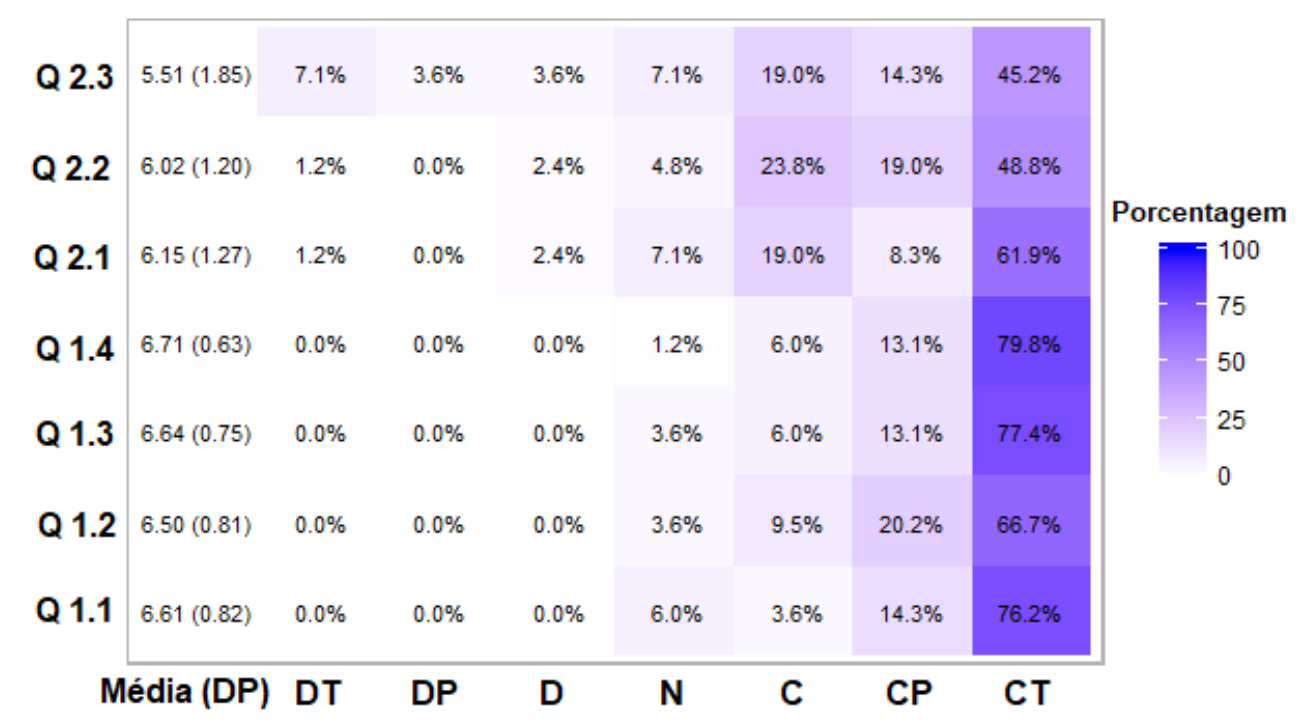

Figura 7. Respostas às questões sobre satisfação na utilização da ferramenta.

Além das questões objetivas (em escala likert), no questionário também havia uma questão para os estudantes relatarem um breve comentário sobre suas impressões sobre a plataforma. Neste caso, a grande maioria das respostas foram positivas Alguns dos comentários apresentados pelos estudantes estão relatados a seguir:

"Muitas vezes eu penso em começar a estudar tal matéria mas não sei por onde começar, com o uso da ferramento ela poderá me auxiliar por onde começar e trazer materiais eficientes para minha aprendizagem, tornando tudo mais fácil e menos confuso..."

"Gostei de utilizar esta ferramenta, pois sinto dificuldades em fazer exercícios sozinha."

"O sistema ficou bem legal e os materiais"

A confiabilidade das respostas objetivas apresentadas no questionário foi verificada com o teste de Alfa de Cronbach ${ }^{2}$. Esse teste analisa a consistência interna das

\footnotetext{
${ }^{2}$ Para interpretação dos valores do Alfa de Cronbach, utilizaram-se os adjetivos propostos por [Landis and Koch 1977], que definem os seguintes intervalos: $\alpha>0.80=$ consistência interna quase perfeita; $0.61<\alpha<0.80=$ consistência interna substancial; $0.41<\alpha<0.60=$ consistência interna moderada; $0.21<\alpha<0.40=$ consistência interna razoável; $\alpha<0.21=$ consistência interna pequena.
} 
respostas com base na correlação entre diferentes itens para uma mesma escala. Neste experimento as questões foram agrupadas em duas categorias: CAT1 para influência da ferramenta na perspectiva de utilidade, e CAT2 para alteração no comportamento de estudo. A Tabela 1 registra os valores de Alfa de Cronbach obtidos.

\begin{tabular}{|c|c|c|c|c|c|}
\hline Categoria & Questão & Mediana & Moda & Erro desvio & $\alpha^{1}$ \\
\hline \multirow{4}{*}{ CAT1 } & Q1.1 & 7.00 & 7 & 0.82 & \multirow{4}{*}{0.752} \\
\hline & Q1.2 & 7.00 & 7 & 0.81 & \\
\hline & Q1.3 & 7.00 & 7 & 0.75 & \\
\hline & Q1.4 & 7.00 & 7 & 0.63 & \\
\hline \multirow{3}{*}{ CAT2 } & $\mathrm{Q} 2.1$ & 7.00 & 7 & 1.26 & \multirow{3}{*}{0.636} \\
\hline & Q2.2 & 6.00 & 7 & 1.20 & \\
\hline & Q2.3 & 6.00 & 7 & 1.84 & \\
\hline
\end{tabular}

${ }^{1}$ Consistência interna medida pelo Alfa de Cronbach.

Verifica-se que, para as duas categorias foram obtidas consistências internas substanciais, o que aponta que as respostas dos estudantes foram muito consistentes e seguiram um padrão bem confiável. Mesmo apresentando uma consistência substancial, é possível observar nos resultados que a CAT1 esteve bem próxima de alcançar a confiabilidade quase perfeita. Por outro lado, a consistência da CAT2 foi bem próxima de uma consistência interna moderada.

\section{Considerações Finais}

Este artigo apresenta a Math Suggestion, uma ferramenta para recomendação de OAs baseada na teoria de crenças de autoeficácia e desempenho. A ferramenta disponibiliza um arcabouço de recursos para que professores criem instrumentos avaliativos para avaliar percepções da capacidade e do desempenho e, a partir das respostas dos estudantes, recomendar conteúdo educacional de acordo com suas capacidades e limitações.

Para análise da proposta foram executados experimentos com estudantes ingressantes de três cursos de graduação. Foi possível constatar que os estudantes aprovaram o uso da ferramenta e se sentiram mais motivados e propícios a uma mudança de comportamento frente a sua utilização. Experimentos também mostraram que os estudantes acreditam que a ferramenta pode contribuir significativamente para o processo de ensino/aprendizagem.

Potenciais trabalhos futuros incluem novas formas de visualização de dados, como skillmeters e mapas de conhecimento, gráficos de área, gráficos de dispersão, implementação de outros algoritmos para recomendação do conteúdo ao estudante, e, por fim, criação de um repositório base de instrumentos avaliativos para diferentes níveis de educação (básico, fundamental, médio e superior).

\section{Agradecimentos}

Os autores agradecem o apoio do IFSULDEMINAS para a realização deste trabalho. 


\section{Referências}

Aguiar, J., Santos, S., Fechine, J., and Costa, E. (2015). Avaliação de sistemas de recomendação educacionais no brasil: uma revisão sistemática da literatura. In Simpósio Brasileiro de Informática na Educação - SBIE, volume 26, page 1255.

Bandura, A. (1986). Social foundations of thought and action. Englewood Cliffs, NJ, 1986.

Bandura, A. (1997). Self-efficacy: The exercise of control. Macmillan.

Barros, A. (1996). Atribuições causais e expectativa de controlo na realização matemática. Psychologica, 15:135-146.

Bray, A. and Tangney, B. (2017). Technology usage in mathematics education research-a systematic review of recent trends. Computers \& Education, 114:255-273.

Brito, M. R. F. d. and Souza, L. F. N. I. d. (2015). Autoeficácia na solução de problemas matemáticos e variáveis relacionadas. Temas em Psicologia, 23(1).

Dobarro, V. R. and Brito, M. R. F. (2010). Atitude e crença de auto-eficácia: relações com o desempenho em matemática. Educação Matemática Pesquisa: Revista do Programa de Estudos Pós-Graduados em Educação Matemática, 12(2).

Dorça, F. A., Araújo, R. D., De Carvalho, V. C., Resende, D. T., and Cattelan, R. G. (2016). An automatic and dynamic approach for personalized recommendation of learning objects considering students learning styles: An experimental analysis. Informatics in education, 15(1):45-62.

Imran, H., Belghis-Zadeh, M., Chang, T.-W., Graf, S., et al. (2016). Plors: a personalized learning object recommender system. Vietnam Journal of Comp. Science, 3(1):3-13.

Landis, J. R. and Koch, G. G. (1977). The measurement of observer agreement for categorical data. Biometrics, 33(1).

Lent, R. W., Brown, S. D., and Larkin, K. C. (1984). Relation of self-efficacy expectations to academic achievement and persistence. Journal of counseling psychology, 31(3):356.

Pajares, F. (1996). Self-efficacy beliefs and mathematical problem-solving of gifted students. Contemporary educational psychology, 21(4):325-344.

Pajares, F. and Miller, M. D. (1994). Role of self-efficacy and self-concept beliefs in mathematical problem solving: A path analysis. Journal of educational psychology, 86(2):193.

Pietsch, J., Walker, R., and Chapman, E. (2003). The relationship among self-concept, self-efficacy, and performance in mathematics during secondary school. Journal of educational psychology, 95(3):589.

Pukkhem, N. (2013). Ontology-based semantic approach for learning object recommendation. International Journal on Information Technology, 3(4):12.

Rezende, P. A., Pereira, C., Campos, F., David, J., and Braga, R. (2015). Personna: proposta de ontologia de contexto e perfil de alunos para recomendação de objetos de aprendizagem. Revista Brasileira de Informática na Educação, 23(01):70. 
Rodrigues, S. d. S. (2017). Eficácia docente no ensino da matemática. Ensaio: Avaliação e Políticas Públicas em Educação, 25(94):114-147.

Syed, T. A., Palade, V., Iqbal, R., and Nair, S. S. K. (2017). A personalized learning recommendation system architecture for learning management system. In KDIR, pages $275-282$.

Tomás da Silva, J., Paula Paixão, M., Sousa Machado, T., and Pacheco Miguel, J. (2017). Rendimento escolar na matemática: Efeito diferencial da ansiedade e das crenças de autoeficácia. Rev. De Est. e Investigación en Psicología Y Educación, (01):057.

Zia, A. and Usman, M. (2018). Elective learning objects group recommendation using non-cooperative game theory. In 2018 International Conference on Frontiers of Information Technology (FIT), pages 194-199. IEEE. 